\title{
STABLE VISCOSITIES AND SHOCK PROFILES FOR SYSTEMS OF CONSERVATION LAWS ${ }^{1}$
}

BY

\author{
ROBERT L. PEGO
}

\begin{abstract}
Wide classes of high order "viscosity" terms are determined, for which small amplitude shock wave solutions of a nonlinear hyperbolic system of conservation laws $u_{t}+f(u)_{x}=0$ are realized as limits of traveling wave solutions of a dissipative system $u_{t}+f(u)_{x}=\nu\left(D_{1} u_{x}\right)_{x}+\cdots+\nu^{n}\left(D_{n} u^{(n)}\right)_{x}$. The set of such "admissible" viscosities includes those for which the dissipative system satisfies a linearized stability condition previously investigated in the case $n=1$ by A. Majda and the author. When $n=1$ we also establish admissibility criteria for singular viscosity matrices $D_{1}(u)$, and apply our results to the compressible Navier-Stokes equations with viscosity and heat conduction, determining minimal conditions on the equation of state which ensure the existence of the "shock layer" for weak shocks.
\end{abstract}

1. Introduction. Consider a hyperbolic system of $m$ conservation laws in one space dimension,

$$
u_{t}+f(u)_{x}=0, \quad u \in \mathbf{R}^{m} .
$$

There are several ways in which higher order systems have been naturally associated with (1.1). Physically, (1.1) often arises as a model for a system with small, high order viscosity and/or dispersion terms. Prototype examples are the compressible Navier-Stokes equations in one space dimension, the KdV equation, or the KdV-Burgers equation $u_{t}=\left(u^{2} / 2\right)_{x}+\nu u_{x x}+\mu u_{x x x}$. Secondly, smooth solutions to the Cauchy problem for (1.1) generally develop discontinuities in finite time, but weak solutions are not unique, so one hopes to identify unique solutions mathematically as limits of solutions of some regularized equation. High order terms may be associated with (1.1) in a third way: given a finite difference approximation to (1.1), it often approximates to better accuracy solutions of an equation with additional dissipative and dispersive terms [5]. Study of that equation may shed light on the behavior of the difference scheme.

What types of higher order equations are appropriate for approximating discontinuous solutions of (1.1)? We consider this model problem: Determine those matrix $n$-tuples $\left(D_{1}, \ldots, D_{n}\right)$ with the following property: A simple discontinuous solution of $(1.1)$ in the form

Received by the editors April 25, 1983.

1980 Mathematics Subject Classification. Primary 35L65, 35L67, 35B99, 35Q10, 76N10, 35K65.

Key words and phrases. Shock profiles, viscosity, traveling waves, center manifold, compressible Navier-Stokes equations, shock layer.

'Sponsored by the U. S. Army under Contract No. DAAG29-80-C-0041. This material is based upon work supported by the National Science Foundation under Grant No. MCS-7927062, Mod. 2. 


$$
u(x, t)= \begin{cases}u_{L}, & x<s t, \\ u_{R}, & x>s t,\end{cases}
$$

is the limit of smooth traveling wave solutions $u^{\nu}=U((x-s t) / \nu)$ of an "approximating" system of higher order,

$$
u_{t}+f(u)_{x}=\nu\left(D_{1} u_{x}\right)_{x}+\cdots+\nu^{n}\left(D_{n} u^{(n)}\right)_{x}
$$

as $\nu \downarrow 0$ if (and only if) the solution (1.2) satisfies a suitable entropy condition (see §3). Such an $n$-tuple is called admissible. A solution (1.2) is called a shock wave if the entropy condition is satisfied. (1.2) is a weak solution of (1.1) precisely when the Rankine-Hugoniot jump conditions are satisfied:

$$
f\left(u_{R}\right)-f\left(u_{L}\right)-s\left(u_{R}-u_{L}\right)=0 .
$$

The traveling wave solution $U((x-s t) / v)$ of (1.3) is called a shock profile. With $\xi=(x-s t) / \nu, U(\xi)$ is required to satisfy the system of ODEs

$$
f(U)-f\left(u_{L}\right)-s\left(U-u_{L}\right)=D_{1} \frac{d U}{d \xi}+\cdots+D_{n} \frac{d^{n} U}{d \xi^{n}}
$$

together with boundary conditions

$$
\begin{gathered}
U(\xi) \rightarrow \begin{cases}u_{L} & \text { as } \xi \rightarrow-\infty, \\
u_{R} & \text { as } \xi \rightarrow \infty, .\end{cases} \\
d^{j} U / d \xi^{j} \rightarrow 0 \quad \text { as } \xi \rightarrow \pm \infty, \quad j=1, \ldots, n-1 .
\end{gathered}
$$

$D_{j}$ may be a smooth function $D_{j}\left(u, u_{x}, \ldots, u^{(n-1)}\right)$ for $j=1, \ldots, n$.

We assume that system (1.1) is strictly hyperbolic, so that if $A(u)=\partial f / \partial u$ is the Jacobian matrix, $A(u)$ has $m$ distinct real eigenvalues, ordered $\lambda_{1}(u)<\cdots<\lambda_{m}(u)$ with corresponding right and left eigenvectors $r_{k}(u)$ and $l_{k}(u), k=1, \ldots, m$, satisfying

$$
\left(A-\lambda_{j}\right) r_{j}(u)=0, \quad\left(A^{*}-\lambda_{k}\right) l_{k}(u)=0, \quad l_{k} \cdot r_{j}=\delta_{k j} .
$$

An eigenvalue $\lambda_{j}(u)$ is called genuinely nonlinear (resp. linearly degenerate) if $\nabla \lambda_{j} \cdot r_{j}(u)$ does not vanish (resp. vanishes identically).

The problem above originated with Gelfand [2], who suggested that the entropy condition singles out those simple discontinuities (1.2) which are limits of traveling wave solutions of parabolic systems associated with (1.1) (the case $n=1$ here). In more concrete form, the investigation of the "shock layer" in gas dynamics dates back much further (see [3]). Most previous work on the problem has been for the case $n=1$; however, Shapiro [9] and Smoller and Shapiro [10] have obtained some results in the case $n=2$, assuming genuine nonlinearity.

The present work is based on the analysis (for the case $n=1$ ) of Majda and Pego [6], who describe a natural algebraic condition on the viscosity matrix $D=D_{1}$ called strict stability, and show that any strictly stable matrix is admissible for all weak shocks $\left(\left|u_{L}-u_{R}\right|\right.$ small). They also obtain conditions which characterize (up to a degenerate class) those matrices admissible for weak $k$-shocks (those associated with a particular $\lambda_{k}$, see $\S 3$ ). 
This paper extends the analysis of [6] in two directions. First, admissibility criteria and a notion of strict stability are developed for $n$-tuples $\left(D_{1}, \ldots, D_{n}\right)$ for any $n(\S \S 2$, 3 ). Second, admissibility criteria are established in the case $n=1$ for singular viscosity matrices $D(u)$, typical in physical systems $(\S 4)$. Indeed, in the last section we apply our results to the compressible Navier-Stokes equations of gas dynamics, determining minimal conditions the equation of state should satisfy to ensure the existence of the shock layer for weak shocks, and to ensure that the stability condition holds.

2. Stable viscosities for strictly hyperbolic systems. Following [6], the notion of stability for an $n$-tuple $\left(D_{1}, \ldots, D_{n}\right)$ may be motivated as follows: Linearize (1.3) at a constant state $u_{0}$, obtaining

$$
u_{t}+A\left(u_{0}\right) u_{x}=\nu D_{1} u_{x x}+\cdots+\nu^{n} D_{n} u^{(n+1)} .
$$

If (1.3) is to be a good approximation to (1.1), a reasonable requirement to be imposed is that the Cauchy problem for $(2.1)$ be strongly well posed, independent of $\nu$ as $\nu \downarrow 0$. In $L^{2}$, using the Fourier transform gives this notion an algebraic interpretation:

Definition. We call the $n$-tuple $\left(D_{1}, \ldots, D_{n}\right)$ stable for $(2.1)$ if for each $T>0$ there exists $C(T)$ such that

$$
\sup _{\substack{0 \leq t \leq T \\ \nu>0 \\ \xi \text { real }}}\left\|\exp t\left(-i \xi A\left(u_{0}\right)-\nu \xi^{2} D_{1}+\cdots+\nu^{n}(i \xi)^{n+1} D_{n}\right)\right\| \leqslant C(T) .
$$

We denote by $S_{n}\left(u_{0}\right)$ the set of stable $n$-tuples, considered topologically as a subset of $\mathbf{R}^{n m^{2}}$. An $n$-tuple in the interior of $S_{n}\left(u_{0}\right)$ is called strictly stable at $u_{0}$.

REMARK. Introduce the matrix polynomial

$$
P(\xi)=-i \xi A\left(u_{0}\right)-\xi^{2} D_{1}+\cdots+(i \xi)^{n+1} D_{n} .
$$

Condition (2.2) is equivalent to

$$
\sup _{\substack{t \geq 0 \\ \zeta \text { real }}}\|\exp t P(\zeta)\| \leqslant C .
$$

This section is devoted to describing the structure of the set of stable $n$-tuples. However, we point out that a major objective of this paper is to prove the following:

THEOREM 2.1. Suppose the $n$-tuple $\left(D_{1}, \ldots, D_{n}\right)$ is strictly stable at $u_{0}$. If $n$ is even, also assume $D_{n}$ is nonsingular. Then $\left(D_{1}, \ldots, D_{n}\right)$ is admtssible for all shocks in some fixed neighborhood $N$ of $u_{0}$. That is, if $u_{L}$ and $u_{R}$ are in $N$ and satisfy (1.4), then $a$ corresponding shock profile solution of (1.5), lying in $N$ and satisfying (1.6), exists if -and only if Liu's strict entropy condition $(\mathrm{E})_{\text {s }}$ (see §3) is satisfied by the jump (1.2).

This theorem is a corollary of Theorems 2.3 and 3.1 below. We state here another corollary of Theorem 2.3, giving a convenient sufficient condition for strict stability:

COROllary 2.2. An n-tuple $\left(D_{1}, \ldots, D_{n}\right)$ is strictly stable at $u_{0}$ if there is a positive definite symmetric matrix $E$ such that $E A\left(u_{0}\right)$ is symmetric and 
(i) $E D_{j}$ is symmetric if $j$ is even,

(ii) $E D_{j} i^{j-1}$ is positive definite if $j$ is odd.

If $n$ is even, we also require that $D_{n}$ has distinct eigenvalues.

The basic result of this section is Theorem 2.3 below, which characterizes strictly stable $n$-tuples. Theorem 2.4 completes the description of the set of stable $n$-tuples for odd $n$. The difficulties encountered in trying to extend that result to even $n$ are analogous to those involved when (1.1) is not strictly hyperbolic.

THEOREM 2.3. The $n$-tuple $\left(D_{1}, \ldots, D_{n}\right)$ is strictly stable at $u_{0}$ if and only if the following conditions hold:

(i) $l_{k} D_{1} r_{k}\left(u_{0}\right)>0, k=1, \ldots, m$.

(ii) If $\xi \neq 0$, then $P(\xi)$ has no purely imaginary eigenvalue.

(iii) (a) If $n$ is odd, the eigenvalues of $D_{n} i^{n-1}$ have positive real part.

(b) If $n$ is even, the eigenvalues of $D_{n}$ are real and distinct, and if $l_{k}^{\infty}$ and $r_{k}^{\infty}$ denote corresponding left and right eigenvectors ( with $l_{i}^{\infty} \cdot r_{j}^{\infty}=\delta_{i j}$ ), then $l_{k}^{\infty} D_{n-1} r_{k}^{\infty} i^{n-2}>0$.

THEOREM 2.4. The set $S_{n}\left(u_{0}\right)$ of stable $n$-tuples is the closure of its interior if $n$ is odd.

In the rest of this section, we prove $2.2-2.4$. We begin by developing necessary criteria for stability. If $(2.3)$ holds, then the eigenvalues of $P(\xi)$ must have nonpositive real part for all real $\xi$. Using this principle, we may establish:

Proposition 2.5. Assume $\left(D_{1}, \ldots, D_{n}\right)$ is stable at $u_{0}$. Then

(i) $l_{k} D_{1} r_{k}\left(u_{0}\right) \geqslant 0, k=1, \ldots, m$.

(ii) For any eigenvalue $\kappa_{j}(\xi)$ of $P(\xi), \operatorname{Re} \kappa_{j}(\xi) \leqslant 0, j=1, \ldots, m$.

(iii) (a) If $n$ is odd, the eigenvalues of $D_{n} i^{n-1}$ have nonnegative real part.

(b) If $n$ is even, the eigenvalues of $D_{n}$ are real, and if they are distinct, then $l_{k}^{\infty} D_{n} r_{k}^{\infty} i^{n-2} \geqslant 0, k=1, \ldots, m$ (notation as in 2.3(iii)(b)).

Proof. (ii) is immediate. For convenience, we define

$$
\begin{aligned}
B(\theta) & =-P(\tan \theta)(\cos \theta)^{n} / \tan \theta \\
& =(\cos \theta)^{n} i A\left(u_{0}\right)+\sin \theta(\cos \theta)^{n-1} D_{1}+\cdots+(\sin \theta)^{n} i^{n-1} D_{n} .
\end{aligned}
$$

From (ii) and continuity the eigenvalues $\mu_{j}(\theta)$ of $B(\theta)$ satisfy

$$
(\sin \theta) \operatorname{Re} \mu_{j}(\theta) \geqslant 0, \quad-\pi / 2 \leqslant \theta \leqslant \pi / 2, \quad j=1, \ldots, m .
$$

Setting $\theta= \pm \pi / 2$ we obtain (iii)(a) and part of (iii)(b). For (i), $B(0)=i A$ has distinct eigenvalues, so for small $\theta$ there exist smooth eigenvalues $\mu_{k}(\theta)$ and eigenvectors $R_{k}(\theta)$, with $\mu_{k}(0)=i \lambda_{k}\left(u_{0}\right), R_{k}(0)=r_{k}\left(u_{0}\right)$, satisfying

$$
\left(B(\theta)-\mu_{k}(\theta)\right) R_{k}(\theta)=0 \text {. }
$$

Differentiate, set $\theta=0$ and dot with $l_{k}\left(u_{0}\right)$, obtaining

$$
l_{k} D_{1} r_{k}\left(u_{0}\right)=\mu_{k}^{\prime}(0)
$$

whence (i). For (iii)(b), a similar procedure played at $\theta=\pi / 2$ yields

$$
-i^{n-2} l_{k}^{\infty} D_{n-1} r_{k}^{\infty}=\mu_{k}^{\prime}(\pi / 2) \text {. }
$$


Proof of 2.3. The necessity of the conditions is easily established for the most part by considering scalar perturbations of $D_{1}, D_{n}, D_{n-1}$ as appropriate. To show that $D_{n}$ must have distinct eigenvalues when $n$ is even, we remark that a Jordan block for a single multiple eigenvalue may be perturbed in the $(1,2)$ and $(2,1)$ positions so as to give rise to complex eigenvalues.

The sufficiency of the conditions is established as for the case $n=1$ in [6], using the Kreiss matrix theorem and the fact that $B(\theta)$ may be smoothly diagonalized for $\theta$ near 0 (and for $\theta$ near $\pm \pi / 2$ if $n$ is even).

Theorem 2.4 follows directly from 2.5 and 2.3. For if $n$ is odd and $\left(D_{1}, \ldots, D_{n}\right)$ is stable, then it is easy to check that $\left(D_{1}+\delta I, D_{2}, \ldots, D_{n-1}, D_{n}+i^{n-1} \delta I\right)$ is strictly stable for any $\delta>0$.

Proof of Corollary 2.2. Observe that if $M$ is any real symmetric matrix, and $z$ a complex vector, then $z^{*} M z$ is real. Also if $M$ is positive definite, but not necessarily symmetric, then $\operatorname{Re}\left(z^{*} M z\right) \geqslant C|z|^{2}$. Now suppose $\left(B(\theta)-\mu_{j}(\theta)\right) z=0$. Then

$$
\operatorname{Re} \mu_{j}(\theta) \cdot z^{*} E z=(\cos \theta)^{n} \sum_{j \text { odd }}(\tan \theta)^{j} \operatorname{Re} z^{*} E D_{j} i^{j-1} z
$$

So for $-\pi / 2<\theta<\pi / 2, \operatorname{Re} \mu_{j}(\theta) \neq 0$, so 2.3(ii) holds. Also, for $\theta>0$ small, $\operatorname{Re} \mu_{j}(\theta) \geqslant C \theta$, so $\mu_{k}^{\prime}(0)=l_{k} D_{1} r_{k}>0$, and (i) holds. Similarly, if $n$ is even, (iii)(b) holds, for then

$$
\begin{aligned}
\operatorname{Re} \mu_{j}(\theta) z^{*} E z & =(\sin \theta)^{n} \sum_{j \text { odd }}(\cot \theta)^{n-j} \operatorname{Re} z^{*} E D_{j} i^{j-1} z \\
& \geqslant C(\pi / 2-\theta) \text { for } \pi / 2-\theta>0 \text { small. }
\end{aligned}
$$

3. Admissibility for weak $k$-shocks. In this section we characterize, up to a degenerate class, those $n$-tuples $\left(D_{1}, \ldots, D_{n}\right)$ which are admissible for weak shocks of a particular family. As in [6], the center manifold theorem is used to find a trajectory connecting critical points in an appropriate system of ODEs.

We begin by defining Liu's strict entropy condition. First consider the structure of the Hugoniot set of solutions of the Rankine-Hugoniot conditions (1.4). Fixing $u_{L}$, the local structure of this set is well known [1]. In some neighborhood of $u_{L}$, the possible solutions $u_{R}$ lie on $m$ curves, $u_{R}=\tilde{u}^{k}(\rho), k=1, \ldots, m$, passing through $u_{L}$ with corresponding shock speeds $s=s^{k}(\rho), k=1, \ldots, m$, satisfying

$$
\begin{array}{ll}
\tilde{u}^{k}(0)=u_{L}, & s^{k}(0)=\lambda_{k}\left(u_{L}\right), \\
\frac{d \tilde{u}^{k}}{d \rho}(0)=r_{k}\left(u_{L}\right), & \frac{d s^{k}}{d \rho}(0)=\frac{1}{2} \nabla \lambda_{k} \cdot r_{k}\left(u_{L}\right), \\
\rho=l_{k}\left(u_{L}\right) \cdot\left(\tilde{u}^{k}(\rho)-u_{L}\right) . &
\end{array}
$$

Liu's strict entropy condition for a $k$-wave (1.2) with $u_{R}=\tilde{u}^{k}\left(\rho_{R}\right)$ is:

$$
s^{k}(\rho)>s=s^{k}\left(\rho_{R}\right) \text { for } \rho \text { between } 0 \text { and } \rho_{R} .
$$

If $\lambda_{K}(u)$ is genuinely nonlinear and $\left|u_{L}-u_{R}\right|$ small, this condition is equivalent to Lax's shock inequalities (see [6]). 
Theorem 3.1. Fix $u_{0} \in \mathbf{R}^{m}$ and $k, 1 \leqslant k \leqslant m$. Assume $\lambda_{k}(u)$ is not linearly degenerate in any neighborhood of $u_{0}$. Assume $\left(D_{1}, \ldots, D_{n}\right)$ satisfies the following nondegeneracy conditions at $u_{0}$ :

(i) $D_{n}$ is nonsingular.

(ii) $l_{k} D_{1} r_{k} \neq 0$.

(iii) $-i \xi\left(A-\lambda_{k}\right)\left(u_{0}\right)-\xi^{2} D_{1}+\cdots+(i \xi)^{n+1} D_{n}$ is nonsingular for all real $\xi \neq 0$.

Then the following are equivalent:

(1) $t_{k} D_{1} r_{k}\left(u_{0}\right)>0$.

(2) $\left(D_{1}, \ldots, D_{n}\right)$ is locally admissible for all $k$-shocks in a neighborhood of $u_{0}$. That is, there exists $\delta>0$ so that if $u_{L}$ and $u_{R}$ in $B_{\delta}\left(u_{0}\right)$ satisfy the jump conditions (1.4) for some $s=s^{k}\left(\rho_{R}\right)$, then a shock profile lying in $B_{\delta}\left(u_{0}\right)$ exists connecting $u_{L}$ to $u_{R}$ if and only if Liu's strict entropy condition $(\mathrm{E})_{s}$ is satisfied.

Theorem 2.1 is an immediate corollary of this theorem, using 2.3. We proceed to the proof of 3.1. Our first step is to rewrite (1.5) as an equivalent first order autonomous system of ODEs. Introduce variables $w^{j}=u^{(j)}$ for $j=0,1, \ldots, n-1$ and introduce the parameters $v=u_{L}$ and $s$ as additional variables. (1.5) is now written, in block form, as

$$
\begin{aligned}
& w_{\xi}^{0}=w^{1} \\
& w_{\xi}^{1}= w^{2} \\
& \vdots \\
& w_{\xi}^{n-1}=D_{n}^{-1}\left(f\left(w^{0}\right)-f(v)-s\left(w^{0}-v\right)-\sum_{j=1}^{n-1} D_{j} w^{j}\right) \\
& v_{\xi}=0 \\
& s_{\xi}=0
\end{aligned}
$$

The existence of a shock profile satisfying (1.6) corresponds to the existence of a trajectory of system (3.2) connecting the critical point $\left(u_{L}, 0 \cdots 0, u_{L}, s\right)$ to the critical point $\left(u_{R}, 0 \cdots 0, u_{L}, s\right)$. Our analysis is based on the description of the center manifold of (3.2) at the critical point $\left(u_{0}, 0 \cdots 0, u_{0}, \lambda_{k}\left(u_{0}\right)\right)$.

Without loss of generality, assume $u_{0}=0, \lambda_{k}\left(u_{0}\right)=0$. For convenience, introduce the column vector $W=\left(w^{0}-v, w^{1}, \ldots, w^{n-1}, v, s\right)$, so that $W^{0}=w^{0}-v$. Then (3.2) is written

$$
W_{\xi}=T(W) .
$$

For the statement of the center manifold theorem, consult [6]. To apply the theorem, it suffices to describe two invariant subspaces for the linearization $d T$ at the critical point 0 : algebraic eigenspaces corresponding to eigenvalues with zero and nonzero real parts, respectively. To calculate these, compute, in block form on $\mathbf{R}^{n m} \times \mathbf{R}^{m} \times \mathbf{R}$

$$
d T(0)=\left[\begin{array}{ccc}
C_{0} & 0 & 0 \\
0 & 0 & 0 \\
0 & 0 & 0
\end{array}\right]
$$


where $C_{0}$ is a block companion matrix,

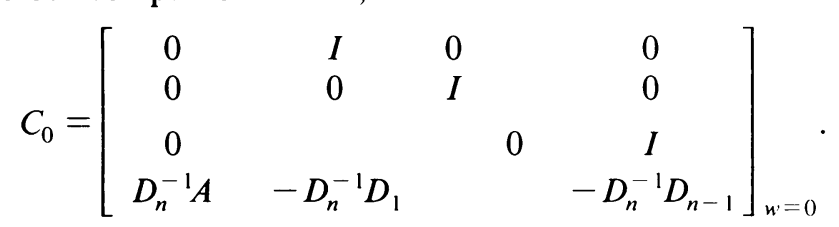

Since det $D_{n} \neq 0$ (and $\lambda_{k}\left(u_{0}\right)=0$ ) the characteristic equation for $d T(0)$ may be written

$$
\lambda^{m+1} \operatorname{det}\left(-A+\lambda D_{1}+\cdots+\lambda^{n} D_{n}\right)=0 .
$$

Condition (iii) of 3.1 guarantees that $d T(0)$ has no nonzero eigenvalues with zero real parts. Condition (ii) of 3.1 guarantees that the zero eigenvalue is semisimple, that is, the algebraic eigenspace for $d T(0)$ for the eigenvalue zero is equal to $\operatorname{ker} d T(0)$. This kernel is spanned by $m+2$ vectors, $\left(R_{k}, 0,0\right),(0,0,1)$, and $\left(0, r_{j}, 0\right), j=1, \ldots, m$. Here $R_{k}=\left(r_{k}, 0 \cdots 0\right) \in R^{n m}$ and $r_{j}=r_{j}(0)$.

Let $Y=\operatorname{ker} d T(0)$ and $X=$ range $d T(0)$. Then $Y$ and $X$ are complementary invariant subspaces corresponding to eigenvalues with zero and nonzero real parts, respectively. Applying the center manifold theorem, we have (see [6]):

Proposition 3.2. Assume $\left(D_{1}, \ldots, D_{n}\right)$ satisfies the nondegeneracy conditions (i)-(iii) at $u_{0}=0$ with $\lambda_{k}\left(u_{0}\right)=0$. Then there exists $\delta>0$ and $a C^{r}$ function $(r \geqslant 2)$ $g: Y \rightarrow X$ defined on $B_{\delta}(0) \cap Y=\{y \in Y|| y \mid<\delta\}$ so that

(1) $M^{*}=\left\{x+y \in \mathbf{R}^{n m+m+1} \mid x=g(y)\right\}$ is a locally invariant manifold for the system (3.3).

(2) $g(0)=0$ and $d g(0)=0$. Thus $M^{*}$ is tangent to $Y$ at 0 .

(3) Any trajectory of (3.3) which lies in $B_{\delta}(0)$ for all $\xi$ lies in $M^{*}$. In particular, critical points of (3.3) in $B_{\delta}(0)$ lie in $M^{*}$.

The connection problem for (3.2) is immediately reduced to one for a scalar equation as follows: Define a line in $Y$ parametrized by $y(\eta)=\left(\eta R_{k}, u_{L}, s\right)$. The curve $W(\eta)=y(\eta)+g(y(\eta))$ lies in $M^{*}$ while $|y(\eta)|<\delta$, and is itself locally invariant for (3.3), because $v$ and $s$ are constant, while $g$, mapping into $X$, is of the form $g(y(\eta))=\left(G\left(\eta, u_{L}, s\right), 0,0\right), G \in \mathbf{R}^{n m}$. Returning from $W$ to the $(w, v, s)$ coordinates of (3.2), we find that the system

$$
\begin{aligned}
& w_{\xi}^{0}=w^{1} \\
& w_{\xi}^{1}=w^{2} \\
& w_{\xi}^{n-1}=D_{n}^{-1}\left(f\left(w^{0}\right)-f\left(u_{L}\right)-s\left(w^{0}-u_{L}\right)-\sum_{j=1}^{n-1} D_{j} w^{j}\right)
\end{aligned}
$$

which we write $w_{\xi}=\tilde{T}(w)$, admits an invariant curve

$$
w\left(\eta, u_{L}, s\right)=\left(u_{L}+\eta r_{k}, 0 \cdots 0\right)+G\left(\eta, u_{L}, s\right)
$$

so long as $|y(\eta)|=\left|\eta r_{k}\right|+\left|u_{L}-u_{0}\right|+\left|s-\lambda_{k}\left(u_{0}\right)\right|<\delta$. It follows from part (3) of the above proposition that the point $\left(u_{R}, 0 \cdots 0\right)$ lies on this invariant curve if $u_{R} \in B_{\delta}\left(u_{0}\right)$ and (1.4) holds. 
The flow on the invariant curve $w\left(\eta, u_{L}, s\right)$ is now determined by a scalar equation for $\eta(\xi)$,

$$
\eta_{\xi}=F\left(\eta, u_{L}, s\right)
$$

where $F$ is determined from the relation

$$
w_{\eta} F\left(\eta, u_{L}, s\right)=\tilde{T}\left(w\left(\eta, u_{L}, s\right)\right) .
$$

The remainder of the proof is an analysis of the connection problem for the scalar equation (3.7). Two critical points $\eta_{L}=0$ and $\eta_{R}$ are connected, left to right, by a trajectory of (3.7) if and only if $\operatorname{sgn} F\left(\eta, u_{L}, s\right)=\operatorname{sgn} \eta_{R}$ for $\eta$ between 0 and $\eta_{R}$. To compute this sign, it helps to look at the derivative $F_{\eta}\left(0, u_{L}, s\right)$. Differentiate (3.8) with respect to $\eta$ and evaluate at $\eta=0$, obtaining

$$
\begin{aligned}
& F_{\eta}\left(0, u_{L}, s\right) w_{\eta}^{j}=w_{\eta}^{j+1}\left(0, u_{L}, s\right) \text { for } j=0, \ldots, n-2, \\
& F_{\eta} D_{n} w_{\eta}^{n-1}=\left(A\left(u_{L}\right)-s\right) w_{\eta}^{0}-\sum_{j=1}^{n-1} D_{j} w_{\eta}^{j} .
\end{aligned}
$$

Use the first $n-1$ equations to eliminate $w_{\eta}^{1}, \ldots, w_{\eta}^{n-1}$ from the last equation and dot with $l_{k}\left(u_{L}\right)$ to obtain

$$
F_{\eta} \sum_{j=1}^{n}\left(F_{\eta}\right)^{j-1} l_{k} D_{j} w_{\eta}^{0}=\left(\lambda_{k}\left(u_{L}\right)-s\right) l_{k} w_{\eta}^{0}\left(0, u_{L}, s\right) .
$$

We assume $\delta$ is so small that for each $u_{L}$ in $B_{\delta}\left(u_{0}\right)$, the Hugoniot curves $\tilde{u}^{k}\left(\rho, u_{L}\right)$ in $B_{\delta}\left(u_{0}\right)$ are described as at the beginning of this section. The invariant curve $w\left(\eta, u_{L}, s\right)$ intersects the Hugoniot curve $\tilde{u}^{k}\left(\rho, u_{L}\right)$ just when $\eta$ is a critical point of (3.7). We will need to define a correspondence between $\eta$ and $\rho$ (given $u_{L}$ and $s$ ) by

$$
\rho(\eta)=l_{k}\left(u_{L}\right)\left(w^{0}\left(\eta, u_{L}, s\right)-u_{L}\right), \quad \text { so } \quad \rho_{\eta}=l_{k}\left(u_{L}\right) w_{\eta}^{0} .
$$

LEMMA 3.3. If $\delta$ is sufficiently small, then if $\left|u_{L}-u_{0}\right|+\left|s-\lambda_{k}\left(u_{0}\right)\right|<\delta$, we have:

(1) $\operatorname{sgn} l_{k}\left(u_{L}\right) D_{1}(u) w_{\eta}^{0}=\operatorname{sgn} l_{k} D r_{k}\left(u_{0}\right)$ and $\operatorname{sgn} l_{k}\left(u_{L}\right) w_{\eta}^{0}=1$ for $w$ in $B_{\delta}\left(u_{0}, 0 \cdots 0\right)$. (Hence $\rho$ increases with $\eta$.)

(2) $F\left(\eta, u_{L}, s\right)=0$ if and only if $s^{k}(\rho(\eta))=s$ or $\eta=0$.

(3) For all $\eta$ between 0 and $\eta_{0}$,

$$
\operatorname{sgn} F\left(\eta, u_{L}, s\right) \operatorname{sgn} l_{k} D_{1} r_{k}\left(u_{0}\right)=\operatorname{sgn} \eta\left(s^{k}(\rho(\eta))-s\right)
$$

provided $s^{k}(\rho(\eta))-s$ is of one sign between 0 and $\eta_{0}$.

Using (3.11) we may complete the proof of Theorem 3.1. Assume $u_{L}$ and $u_{R}=\tilde{u}^{k}\left(\rho_{R}, u_{L}\right)$ satisfy the Rankine-Hugoniot relations with $s=s^{k}\left(\rho_{R}\right)$, and assume that Liu's strict entropy condition (E) $)_{s}$ holds. Then $u_{R}=w^{0}\left(\eta_{R}, u_{L}, s\right)$ for some $\eta_{R}$, and $\rho_{R}=l_{k}\left(u_{L}\right)\left(u_{R}-u_{L}\right)=\rho\left(\eta_{R}\right)$. By (3.11) and condition $(\mathrm{E})_{s}$,

$$
\operatorname{sgn} F\left(\eta, u_{L}, s\right) \operatorname{sgn} l_{k} D_{1} r_{k}\left(u_{0}\right)=\operatorname{sgn} \eta_{R}
$$

for all $\eta$ between 0 and $\eta_{R}$. So a trajectory of (3.6) connects $\eta_{L}=0$ and $\eta_{R}$, left to right, if and only if $l_{k} D_{1} r_{k}\left(u_{0}\right)>0$.

If $l_{k} D_{1} r_{k}\left(u_{0}\right)>0$ and $u_{R}$ is as above, but the entropy condition is not satisfied, then either $s^{k}(\rho)=s$ for some $\rho$ between 0 and $\rho_{R}$, whence a critical point separates 
0 and $\eta_{R}$ in (3.8), or else $s^{k}(\rho)<s$ for all $\rho$ between 0 and $\rho_{R}$. Then (3.8) implies that a trajectory of (3.5) connects $\eta_{R}$ on the left to $\eta_{L}=0$ on the right. In either case, no trajectory of (1.6) lying in $B_{\delta}\left(u_{0}, 0 \cdots 0\right)$ can connect $u_{L}$ on the left to $u_{R}$ on the right.

Proof of Lemma 3.3. Part (1) follows from continuity and the fact that $w_{\eta}^{0}\left(0, u_{0}, \lambda_{k}\left(u_{0}\right)\right)=r_{k}$ since $d G\left(0, u_{0}, \lambda\left(u_{0}\right)\right)=0$. For part (2), if $\delta$ is small and $\eta \neq 0$, then $F\left(\eta, u_{L}, s\right)=0$ if and only if $w^{0}\left(\eta, u_{L}, s\right)$ lies on the $k$ th Hugoniot curve for $u_{L}$, so $w^{0}\left(\eta, u_{L}, s\right)=\tilde{u}^{k}\left(\rho, u_{L}\right)$ for some $\rho$, and $s^{k}(\rho)=s$. But then by (3.1),

$$
\rho=l_{k}\left(u_{L}\right)\left(\tilde{u}^{k}(\rho)-u_{L}\right)=\rho(\eta) .
$$

We shall establish (3) in the case that $s^{k}(\rho(\eta))>s$ for $\eta$ between 0 and $\eta_{0}>0$, and $l_{k} D_{1} r_{k}\left(u_{0}\right)>0$ (remaining cases are similar). First, $\lambda_{k}\left(u_{L}\right)=s^{k}(0) \geqslant s$. Then $\lambda_{k}\left(u_{L}\right)>\tilde{s}$ for any $\tilde{s}<s$. Now from (3.9) and (3.6), $F_{\eta}\left(0, u_{L}, \tilde{s}\right)=O(\delta)$. Therefore, from part (1) and (3.10), $F_{\eta}\left(0, u_{L}, \tilde{s}\right)>0$. If $\tilde{s}$ is close to $s$, then $\rho\left(\eta_{0} / 2, u_{L}, \tilde{s}\right)<$ $\rho\left(\eta_{0}, u_{L}, s\right)$, so $F\left(\eta, u_{L}, \tilde{s}\right)>0$ for $\eta$ between 0 and $\eta_{0} / 2$. (Since $s^{k}\left(\rho\left(\eta, u_{L}, \tilde{s}\right)\right)>\tilde{s}$, $F$ cannot vanish by part (2).) Letting $\tilde{s}$ increase to $s$, we get $F\left(\eta, u_{L}, s\right)>0$ for $\eta$ between 0 and $\eta_{0} / 2$. (Again, $F\left(\eta, u_{L}, s\right)$ cannot vanish between 0 and $\eta_{0}$ by part (2).)

4. Admissibility for singular viscosity matrices. As mentioned in the introduction, viscosity matrices in physical systems are usually singular. In this section we establish quite general admissibility criteria for weak $k$-shocks for such singular viscosity matrices $D(u)$ (in the case $n=1$ ). Our result will be applied in the next section in a physical example, the compressible Navier-Stokes equations.

In the case $n=1$, with $D=D_{1}(u)$, shock profile $U(\xi)$ must satisfy the system

$$
D(U) U_{\xi}=f(U)-f\left(u_{L}\right)-s\left(U-u_{L}\right)
$$

and boundary conditions

$$
U(\xi) \rightarrow u_{L} \quad \text { as } \xi \rightarrow-\infty, \quad U(\xi) \rightarrow u_{R} \quad \text { as } \xi \rightarrow \infty .
$$

Theorem 4.1. Fix $u_{0} \in \mathbf{R}^{m}$ and $k, 1 \leqslant k \leqslant m$. Assume $\lambda_{k}(u)$ is not linearly degenerate in any neighborhood of $u_{0}$. Assume the viscosity matrix $D=D_{1}(u)$ satisfies the following conditions:

(i) $D(u)$ has constant rank in a neighborhood of $u_{0}$.

(ii) $l_{k} D r_{k}\left(u_{0}\right)=0$.

(iii) For all real $\tau$, the matrix $\left[i \tau\left(A-\lambda_{k}\right)-D\right]\left(u_{0}\right)$ is one-to-one on the subspace $\mathrm{C} \cdot Z_{2}$, where

$$
Z_{2}=\left\{v \in R^{m} \mid\left(A-\lambda_{k}\right)\left(u_{0}\right) v \in \text { range } D\left(u_{0}\right)\right\}
$$

Then the following are equivalent:

(1) $l_{k} D r_{k}\left(u_{0}\right)>0$.

(2) $D$ is locally admissible for all $k$-shocks in a neighborhood of $u_{0}$. That is, there exists $\delta>0$ so that if $u_{L}$ and $u_{R}$ in $B_{\delta}\left(u_{0}\right)$ satisfy the jump conditions (1.4) for some $s=s^{k}\left(\rho_{R}\right)$, then a shock profile lying in $B_{\delta}\left(u_{0}\right)$ exists connecting $u_{L}$ to $u_{R}$ if and only if Liu's strict entropy condition $(\mathrm{E})_{s}$ is satisfied. 
The main steps in the proof of this theorem are the same as those for Theorem 3.1 above or Theorem 3.1 of [6]. The difference is that it is a more delicate matter to obtain an autonomous system, like (3.2), to which the center manifold theorem may be applied. Our approach is to use an algebraic condition implied by (4.1) when $D$ is singular to eliminate some variables, then obtain an autonomous system for the remaining variables.

As before, introduce $v=u_{L}$ and $s$ as variables, writing (4.1) as

$$
\begin{gathered}
D(U) U_{\xi}=f(U)-f(v)-s(U-v), \\
v_{\xi}=0, \quad s_{\xi}=0 .
\end{gathered}
$$

We motivate our elimination procedure in the case that $D$ is constant, $f$ linear and $s=\lambda_{k}\left(u_{0}\right)$. Then $(4.2 \mathrm{i})$ is consistent only if $P(A-s)(U-v)=0$ where $P$ is a projection with $\operatorname{ker} P=$ range $D$. Write $U=w+v$ for $w$ in $\operatorname{ker} P(A-s)$. In order to reduce $(4.2 \mathrm{i})$ to an equation for $w_{\xi}$, we should require that $D: \operatorname{ker} P(A-s) \rightarrow$ range $D$ be one-to-one. Note that this entails $D r_{k} \neq 0$ and $l_{k} D \neq 0$, for

$$
\operatorname{dim} \operatorname{ker} P(A-s)=\operatorname{rank} D .
$$

Returning to the case at hand, without loss of generality we assume $u_{0}=0$, $\lambda_{k}\left(u_{0}\right)=0$. Let $Z_{1}=$ range $D(0)$. Recall that $D(0)$ is one-to-one on $Z_{2}$ from (iii), so $\operatorname{dim} Z_{2}=\operatorname{dim} Z_{1}=b$. We may choose (inductively on dimension) a subspace $Z_{3}$ complementary to both $Z_{1}$ and $Z_{2}$, with $\operatorname{dim} Z_{3}=m-b$. For $u$ sufficiently small, we may choose a smooth projection $P(u)$ with range $Z_{3}$ and kernel $\operatorname{range}(D(u))$; note that ker $P A(0)=Z_{2}$.

Given $(U, v, s)$ in $\mathbf{R}^{2 m+1}$, write $U=u^{3}+u^{2}+v$, where $u^{3}$ is in $Z_{3}$ and $u^{2}$ in $Z_{2}$. We seek to express $u^{3}$ as a function of $\left(u^{2}, v, s\right)$, using the consistency criterion for (4.2i):

$$
P(U)(f(U)-f(v)-s(U-v))=0 .
$$

We find it convenient to introduce isomorphisms $I_{2}: \mathbf{R}^{b} \rightarrow Z_{2}, I_{3}: \mathbf{R}^{m-b} \rightarrow Z_{3}$ and to write $u^{2}=I_{2} w, u^{3}=I_{3} \tilde{w}$. Then we can apply $I_{3}^{-1}$ to (4.3), writing $h(\tilde{w}, w, v, s)=0$, where $h: \mathbf{R}^{m-b} \times \mathbf{R}^{b} \times \mathbf{R}^{m} \times \mathbf{R} \rightarrow \mathbf{R}^{m-b}$. In block form, the Jacobian matrix of $h$ at $(\tilde{w}, w, v, s)=0$ is $d h(0)=\left[I_{3}^{-1} P A(0) I_{3}, 0,0,0\right]$ since $\operatorname{ker} P A(0)=Z_{2}$. The first component is an isomorphism on $\mathbf{R}^{b}$, since $P A(0)$ is one-to-one on $Z_{3}$ to itself. Thus the implicit function theorem applies, so that in a neighborhood of 0 we may write $\tilde{w}=\tilde{w}(w, v, s)$, and indeed the total derivative $d \tilde{w}(0)=[0,0,0]$. We may express

$$
U(w, v, s)=I_{3} \tilde{w}(w, v, s)+I_{2} w+v
$$

and replace $(4.2 \mathrm{i})$ by the equation

$$
D \frac{\partial U}{\partial w^{\prime}}(w, v, s) w_{\xi}=f(U(w, v, s))-f(v)-s(U(w, v, s)-v) .
$$

By construction, the right-hand side lies in range $(D(U))$. The matrix $D(\partial U / \partial w)$ : $\mathbf{R}^{b} \rightarrow$ range $D$ is an isomorphism at $(w, v, s)=0$, so also in a neighborhood, since $D$ has constant rank. We may find a smooth generalized inverse $(D(\partial U / \partial w))^{\dagger}(w, v, s)$ : $\mathbf{R}^{m} \rightarrow \mathbf{R}^{b}$ so $(D(\partial U / \partial w))^{\dagger}(D(\partial U / \partial w))=I$ in $\mathbf{R}^{b}$. We have reduced (4.2) to an 
autonomous system in $\mathbf{R}^{b} \times \mathbf{R}^{m} \times \mathbf{R}$ :

$$
\begin{gathered}
w_{\xi}=\left(D \frac{\partial U}{\partial w}\right)^{\dagger}(f(U(w, v, s))-f(v)-s(U(w, v, s)-v)), \\
v_{\xi}=0, \quad s_{\xi}=0,
\end{gathered}
$$

where we write $W_{\xi}=T(W)$ for the variable $W=(w, v, s)$.

We proceed to apply the center manifold theorem to (4.4) at $W=0$. In block form, since $(\partial U / \partial w)(0)=I_{2}$ and $(\partial U / \partial v)(0)=I$ in $\mathbf{R}^{m}$, we have

$$
d T(0)=\left[\begin{array}{ccc}
\left(D I_{2}\right)^{\dagger} A(0) I_{2} & 0 & 0 \\
0 & 0 & 0 \\
0 & 0 & 0
\end{array}\right]
$$

Condition (iii) of the theorem implies that $d T(0)$ has no nonzero imaginary eigenvalues. Condition (ii) implies that the eigenvalue 0 is semisimple. The kernel of $d T(0)$ is spanned by $m+2$ vectors $(0,0,1),\left(0, r_{j}, 0\right), j=1, \ldots, m$, and $(R, 0,0)$, where $I_{2} R=r_{k}$.

So, defining invariant subspaces $X=$ range $d T(0), Y=\operatorname{ker} d T(0)$, we apply the center manifold theorem as in $\$ 3$ to obtain

Proposition 4.2. Assume $D(u)$ satisfies conditions (i)-(iii). Then there exist $\delta>0$ and a $C^{r}$ function $(r \geqslant 2) g: Y \rightarrow X$ defined on $B_{\delta}(0) \cap Y$ in $\mathbf{R}^{b+m+1}$ so that:

(1) $M^{*}=\left\{x+y \in \mathbf{R}^{b+m+1} \mid x=g(y)\right\}$ is a locally invariant manifold for (4.4).

(2) $g(0)=0$ and $d g(0)=0$. Thus $M^{*}$ is tangent to $Y$ at 0 .

(3) Any trajectory of (4.4) which lies in $B_{\delta}(0)$ for all $\xi$ lies in $M^{*}$.

As in $\S 3$, the connection problem for (4.1) is immediately reduced to one for a scalar equation: Define a line in $Y$ by $y(\eta)=\left(\eta R, u_{L}, s\right)$. The curve $W(\eta)=y(\eta)$ $+g(y(\eta))$ is locally invariant for (4.4), meaning it is composed of solution curves. The curve $U(W(\eta))$ is then composed of solution curves of (4.1). We may write this curve as

where

$$
U\left(\eta, u_{L}, s\right)=u_{L}+\eta r_{k}(0)+G\left(\eta, u_{L}, s\right)
$$

$$
G=I_{3} \tilde{w}\left(\eta R+g(y(\eta)), u_{L}, s\right)+I_{2} g(y(\eta)) .
$$

Note that $G(0)=0, d G(0)=0$.

If $u_{R}$ is in $B_{\delta}(0)$, and satisfies (1.4), then $u_{R}=U\left(w_{R}, u_{L}, s\right)$ for some $w_{R}$, and $\left(w_{R}, u_{L}, s\right)$ is a critical point of $T(W)$, so lies in $M^{*}$, hence on $W(\eta)$. Thus for some $\eta_{R}$

$$
u_{R}=u_{L}+\eta_{R} r_{k}+G\left(\eta_{R}, u_{L}, s\right) .
$$

The flow on the invariant curve $U\left(\eta, u_{L}, s\right)$ is determined by a scalar equation

where

$$
\eta_{\xi}=F\left(\eta, u_{L}, s\right) \text {, }
$$

$$
D(U) U_{\eta} F\left(\eta, u_{L}, s\right)=f(U)-f\left(u_{L}\right)-s\left(U-u_{L}\right) .
$$

The remainder of the proof is identical to that for nonsingular $D(u)$ and may be found in [6]. 
5. Weak shock layers in compressible fluid dynamics. Here we use Theorem 4.1 to obtain very weak conditions on the equation of state in the compressible NavierStokes equations in one space dimension which guarantee the existence of weak shock profiles. We also make a brief remark concerning the linearized stability of these equations.

In Lagrangian coordinates, the equations are written in conservation form as

$$
\begin{aligned}
& \tau_{t}-v_{x}=0, \\
& v_{t}+p_{x}=\left(\frac{\mu}{\tau} v_{x}\right)_{x} \\
& \varepsilon_{t}+(p v)_{x}=\left(\frac{\mu v}{\tau} v_{x}\right)_{x}+\left(\frac{\kappa}{\tau} \theta_{x}\right)_{x} .
\end{aligned}
$$

Here $x$ is the Lagrangian mass coordinate, $t$ is time, $\tau$ is specific volume, $v$ is velocity, $p$ is pressure, $\theta$ is temperature, $\mathcal{E}$ is energy density per unit mass, and $\mu$ and $\kappa$ are, respectively, the coefficients of viscosity and heat conduction. $\varepsilon=e+v^{2} / 2$, where $e$ is the internal energy per unit mass. We assume $\tau$ and $\theta$ determine the thermodynamic state of the material, and $e$ and $p$ are given by sufficiently smooth equations of state, $e=e(\tau, \theta), p=p(\tau, \theta) . \mu$ and $\kappa$ are positive, and may also depend smoothly on $\tau$ and $\theta . \tau, \theta$, and $p$ are positive.

We assume that the specific heat at constant volume is a positive function:

$$
c(\tau, \theta)=e_{\theta}(\tau, \theta)>0 .
$$

So $\theta=\theta(\tau, e)$, and with $u=(\tau, v, \mathcal{E}),(5.1)$ may be written in the form

$$
u_{t}+f(u)_{x}=\left(D(u) u_{x}\right)_{x} \text {. }
$$

We shall see presently that the equation $u_{t}+f(u)_{x}=0$ with diffusion of heat and momentum neglected $(\mu=\kappa=0)$ is strictly hyperbolic if and only if

$$
0<-d p /\left.d \tau\right|_{S \text { constant }} \equiv \alpha^{2} \text {. }
$$

Here $S$ denotes the entropy, and $S=S(\tau, \theta)$. This function is related to $e$ and $p$ through the Gibbs relation

$$
\theta d S=d e+p d \tau
$$

Our main result below is that no additional conditions are required to ensure the existence of shock profiles for weak shocks. (The situation is different for stronger shocks; see [8].)

THEOREM 5.1. Fix $u_{0}=\left(\tau_{0}, v_{0}, \mathcal{E}_{0}\right) \in R^{3}, \tau_{0}>0$, and assume (5.2) and (5.4) hold at $u_{0}$. Then there exists $\delta>0$ so that if $u_{L}, u_{R}$ and s satisfy the Rankine-Hugoniot conditions, (1.4) with $u_{L}, u_{R}$ in $B_{\delta}\left(u_{0}\right)=\left\{u|| u-u_{0} \mid<\delta\right\}$, then a shock profile solution $u(x-s t)$ of $(5.3)$ lying in $B_{\delta}\left(u_{0}\right)$ exists connecting $u_{L}$ to $u_{R}$ if and only if Liu's strict entropy condition $(\mathrm{E})_{s}$ is satisfied.

The study of the "shock layer" in compressible fluid dynamics has a long history. Most relevant here are the results of Gilbarg [3] and Liu [4]. Gilbarg established the existence of shock profiles for shocks of any magnitude under two additional 
conditions on the equation of state:

(5.6) $0<d^{2} p /\left.d \tau^{2}\right|_{s \text { constant }}$.

(5.7) $p_{\theta}(\tau, \theta)>0$.

The convexity condition (5.6) implies that the eigenvalues $\lambda_{1}=-\alpha$ and $\lambda_{3}=\alpha$ are genuinely nonlinear. $\left(\lambda_{2}=0\right.$ is linearly degenerate, see below.) In that case the entropy condition has a simple form. Liu introduced an entropy condition appropriate for the nongenuinely nonlinear case, and showed that, with no heat conduction $(\kappa=0)$, shock profiles exist for discontinuities satisfying his entropy condition (see §3). This result holds for strong shocks so long as the Hugoniot curves (see (3.1)) remain regular.

Theorem 5.1 is proved by verifying conditions (i)-(iii) and (1) of Theorem 4.1 for the first and third wave fields $(k=1$ and 3$)$. Discontinuities associated with the second wave field, called contact discontinuities, cannot satisfy the strict entropy condition $(\mathrm{E})_{s}$. The Jacobian of $f(u)$ takes the following form (with $p(u)=$ $\left.p\left(\tau, \theta\left(\tau, \mathcal{E}-v^{2} / 2\right)\right)\right)$

$$
A(\tau, v, \mathcal{E})=\left[\begin{array}{ccc}
0 & -1 & 0 \\
p_{\tau}(u) & p_{v}(u) & p_{\mathscr{E}}(u) \\
v p_{\tau}(u) & p+v p_{v}(u) & v p_{\mathscr{E}}(u)
\end{array}\right]
$$

The viscosity matrix is

$$
\tau D(\tau, v, \mathcal{E})=\left[\begin{array}{ccc}
0 & 0 & 0 \\
0 & \mu & 0 \\
-\mu e_{\tau}(\tau, \theta) & (\mu-\lambda) v & \lambda
\end{array}\right]
$$

where $\lambda=\kappa / c>0$. The information we need will be computed after performing a convenient change of basis (simultaneous similarity transformation of $A$ and $D$ ). First, note that

$$
p_{\tau}(u)=p_{\tau}(\tau, e), \quad p_{\mathcal{E}}(u)=p_{e}(\tau, e), \quad p_{v}(u)=-v p_{e}(\tau, e)
$$

With

$$
T=\left[\begin{array}{ccc}
1 & 0 & 0 \\
0 & 1 & 0 \\
e_{\tau}(\tau, \theta) & v & 1
\end{array}\right]
$$

it follows that $D_{1}=T^{-1} \tau D T=\operatorname{diag}(0, \mu, \lambda)$ and

$$
A_{1}=T^{-1} A T=\left[\begin{array}{ccc}
0 & -1 & 0 \\
p_{\tau}(\tau, e)+p_{e} e_{\tau}(\tau, \theta) & 0 & p_{e}(\tau, e) \\
0 & p+e_{\tau}(\tau, \theta) & 0
\end{array}\right]
$$

The eigenvalues of $A_{1}$ are $-\alpha, 0$, and $\alpha$. To see this, compute

$$
-\alpha^{2}=p(\tau, e(\tau, S))_{\tau}=p_{\tau}(\tau, e)+p_{e}(\tau, e) e_{\tau}(\tau, S)
$$

and observe that $e_{\tau}(\tau, S)=-p$ from (5.5). 
Condition (i) of 4.1 is obviously satisfied. We turn to condition (ii). A matrix $R=\left(r_{1}, r_{2}, r_{3}\right)$ of right eigenvectors of $A_{1}$ is

$$
R=\left[\begin{array}{ccc}
-1 & p_{e} & -1 \\
-\alpha & 0 & \alpha \\
p+e_{\tau} & -\left(p_{\tau}+p_{e} e_{\tau}\right) & p+e_{\tau}
\end{array}\right]
$$

Thus $A_{1} R=R \operatorname{diag}(-\alpha, 0, \alpha)$ and the corresponding matrix of left eigenvectors is $L=R^{-1}$,

$$
L=\frac{1}{2 \alpha^{2}}\left[\begin{array}{ccc}
\left(p_{\tau}+p_{e} e_{\tau}\right) & -\alpha & p_{e} \\
2\left(p+e_{\tau}\right) & 0 & 2 \\
\left(p_{\tau}+p_{e} e_{\tau}\right) & \alpha & p_{e}
\end{array}\right]=\left[\begin{array}{c}
l_{1} \\
l_{2} \\
l_{3}
\end{array}\right]
$$

We compute

$$
l_{1} D_{1} r_{1}=l_{3} D_{1} r_{3}=\left(\mu \alpha^{2}+\lambda p_{e}(\tau, e)\left(p+e_{\tau}(\tau, \theta)\right)\right) / 2 \alpha^{2} .
$$

From (5.5) and the equality $S_{\tau \theta}=S_{\theta \tau}$ one may verify that thermodynamic identity

$$
\theta p_{\theta}(\tau, \theta)=p+e_{\tau}(\tau, \theta) \text {. }
$$

Also, $p_{\theta}(\tau, \theta)=p_{e}(\tau, e) c$, so

$$
l_{1} D_{1} r_{1}=l_{3} D_{1} r_{3}=\left(\mu \alpha^{2}+\lambda \theta c p_{e}^{2}\right) / 2 \alpha^{2}>0
$$

and $4.1(1)$ is satisfied. For later reference, we compute

$$
\alpha^{2} l_{2} D_{1} r_{2}=-\lambda\left(p_{\tau}(\tau, e)+p_{e}(\tau, e) e_{\tau}(\tau, \theta)\right)=-\lambda p_{\tau}(\tau, \theta) .
$$

It remains to verify condition (iii) of 4.1 for $k=1$ and 3. Take $k=1$.

$$
Z_{2}=\left\{u \in \mathbf{R}^{3} \mid \alpha u_{1}=u_{2}\right\}=\left\{u \in \mathbf{R}^{3} \mid\left(A_{1}+\alpha\right) u \in \text { range } D_{1}\right\} .
$$

To show $i \tau\left(A_{1}+\alpha\right)+D_{1}$ is one-to-one on $\mathrm{CZ}_{2}$, it suffices to show (cf. (5.10)) that

$$
\left[i \tau\left(\begin{array}{cc}
p_{\tau}(\tau, \theta)+\alpha^{2} & p_{e} \\
\alpha \theta c p_{e} & \alpha
\end{array}\right)+\left(\begin{array}{cc}
\mu \alpha & 0 \\
0 & \lambda
\end{array}\right)\right]\left(\begin{array}{l}
1 \\
z
\end{array}\right) \neq 0
$$

for any complex $z$. A calculation similar to (5.8), using (5.9), yields

$$
p_{\tau}(\tau, S)=p_{\tau}(\tau, \theta)-\theta c p_{e}^{2}(\tau, e) .
$$

Therefore, multiplying by $\operatorname{diag}(1,1 / \alpha)$ from the left and $\operatorname{diag}(1 / \theta c, 1)$ from the right, it suffices to show that $i \tau A_{2}+D_{2}$ is nonsingular for any $\tau$, where

$$
A_{2}=\left(\begin{array}{cc}
p_{e}^{2} & p_{e} \\
p_{e} & 1
\end{array}\right), \quad D_{2}=\left(\begin{array}{cc}
\alpha \mu / \theta c & 0 \\
0 & \lambda / \alpha
\end{array}\right) .
$$

But $D_{2}$ is positive definite and $A_{2}$ symmetric, so this is true. So 4.1(iii) holds for $k=1$. For $k=3$, replace $-\alpha$ by $+\alpha$ in the above argument. This finishes the proof of Theorem 5.1.

We conclude with a brief remark concerning the linearized stability condition (2.3), where $P(\xi)=-i \xi A-\xi^{2} D$. From Proposition 2.5 and (5.10), a necessary condition for $(2.3)$ to hold is that $p_{\tau}(\tau, \theta) \leqslant 0$. The nondegenerate condition

$$
0<-p_{\tau}(\tau, \theta)
$$


is stronger than (5.4) by (5.11). In fact, (5.2) and (5.12) are sufficient to imply the linearized stability condition, a fact used by Matsumura and Nishida in [7] to establish the full nonlinear stability of the constant state for the compressible Navier-Stokes equations in three space dimensions. A proof that the linearized stability condition holds is similar to the proof, given in [6], of Theorem 2.3 for $n=1$.

ACKnOWledgement. I am grateful for many discussions with Professor Andrew Majda. Most of the present work appears in my thesis, written under his direction at the University of California, Berkeley.

\section{REFERENCES}

1. J. Conlon, $A$ theorem in ordinary differential equations with an application to hyperbolic conservation laws, Adv. in Math. 35 (1980), 1-18.

2. I. M. Gelfand, Some problems in the theory of quasilinear equations, Uspehi Mat. Nauk 14 (1959), 87-158; English transl., Amer. Math. Soc. Transl. (2) 29 (1963).

3. D. Gilbarg, The existence and limit behavior of the one-dimensional shock layer, Amer. J. Math. 7 (1951), 256-274.

4. T. P. Liu, The entropy condition and the admissibility of shocks, J. Math. Anal. Appl. 53 (1976), $78-88$.

5. A. Majda and S. Osher, A systematic approach for correcting nonlinear instabilities, Numer. Math. 30 (1978), 429-452.

6. A. Majda and R. Pego, Stable viscosity matrices for systems of conservation laws, J. Differential Equations (to appear).

7. A. Matsumura and T. Nishida, The initial value problem for the equations of motion of compressible viscous and heat-conductive fluids, Proc. Japan Acad. Ser. A Math. Sci. 55 (1979), 337-341.

8. R. Pego, Nonexistence of a shock layer in gas dynamics with a nonconvex equation of state, Arch. Rational Mech. Anal. (to appear).

9. R. Shapiro, Shock waves as limits of progressive wave solutions of higher order equations, Ph. D. thesis, Univ. of Michigan, 1973.

10. J. Smoller and R. Shapiro, Dispersion and shock wave structure, J. Differential Equations 44 (1982), 281-305.

Mathematics Research Center, University of Wisconsin, Madison, Wisconsin 53706

Current address: Department of Mathematics, University of Michigan, Ann Arbor, Michigan 48109 